\title{
En attendant le vote « pour ». Réflexions sur les campagnes électorales roumaines
}

While waiting for the Vote " for ». Reflexions on the rumanian election

Campaigns

Paul Dobrescu

\section{(2) OpenEdition}

Journals

Édition électronique

URL : http://journals.openedition.org/questionsdecommunication/5691

DOI : 10.4000/questionsdecommunication.5691

ISSN : 2259-8901

Éditeur

Presses universitaires de Lorraine

Édition imprimée

Date de publication : 1 décembre 2003

Pagination : 301-312

ISSN : 1633-5961

Référence électronique

Paul Dobrescu, «En attendant le vote "pour ». Réflexions sur les campagnes électorales roumaines », Questions de communication [En ligne], 4 | 2003, mis en ligne le 23 mai 2012, consulté le 19 avril 2019. URL : http://journals.openedition.org/questionsdecommunication/5691 ; DOI : 10.4000/ questionsdecommunication.5691 


\section{$>$ NOTES DE RECHERCHE}

PAUL DOBRESCU

École nationale d'études politiques et administratives

Université de Bucarest

paul.dobrescu@comunicare.ro

\section{EN ATTENDANT LE VOTE «POUR ». RÉFLEXIONS SUR LES CAMPAGNES ÉLECTORALES ROUMAINES}

Résumé. - Analysant les quatre campagnes électorales que les Roumains ont connues pendant ces dix dernières années, l'auteur évalue l'influence des médias sur l'évolution de l'opinion publique. Le rôle des médias ne peut être envisagé qu'en tenant compte des dimensions temporelle et contextuelle associées à des facteurs propres à chaque électeur : ressources personnelles, familiales, sociales ou économiques. Constatant que lorsque la confrontation entre candidats est serrée, le rôle des médias croit, l'auteur distingue chaque situation électorale. Ainsi, la campagne de 1996 voit-elle l'action décisive des médias, ceux-ci s'imposant comme distributeurs « d'opinions légitimes ». Par ailleurs, le contexte économique difficile a conduit les Roumains à voter, en 1996 et en 2000, contre ceux qui auraient gouverné jusqu'alors. S'est imposé un vote exprimant la souffrance en même temps que la capacité de l'opinion publique à évoluer : un constat qui modifie sensiblement l'hypothèse de la maléabilité de cette dernière.

Mots clés. - Campagnes électorales roumaines, électorat, opinion publique, médias, effets des médias, communication politique. 
$L$ a stabilité ou la mobilité de l'électorat ont été étudiées d'un point de vue théorique, selon une relation de type cause à effet, en tenant compte de l'influence des médias. D'un côté, l'approche empirique traditionnelle de l'étude des effets des médias (représentée par les membres de l'École de Francfort) soutient, par le biais du concept « d'industrie culturelle », l'idée selon laquelle les médias peuvent transformer de vastes audiences. D'un autre coté, l'approche lazarsfeldienne selon laquelle les médias peuvent être puissants, mais sous certaines conditions - effets indirects -, sur certains segments du public - effets limités - (Katz, 1990). Selon Paul Lazarsfeld, l'option finale du vote reflète plus la position sociale que l'exposition aux médias. Pourtant, dans les sociétés démocratiques contemporaines, les électorats ne sont plus aussi stables que l'électorat américain des années 40, étudié par le sociologue. L'exposition aux messages politiques médiatiques représente, tant dans les campagnes électorales qu'en dehors d'elles, une forme institutionnelle de participation politique des citoyens électeurs, une modalité de participation à la vie publique et, finalement, par le biais du vote, aux décisions concernant la société (Almond, Verba, 1996). L'exposition aux médias, comprise comme un suivi conscient et souhaité des messages transmis par les médias, est, selon Sidney Verba, la première unité de mesure de la communication politique d'un pays, une forme passive de participation politique des citoyens. Mais les facteurs de l'exposition aux médias sont différents, puisque peuvent jouer : les ressources personnelles, familiales, sociales ou économiques de chaque électeur. L'un des facteurs les plus importants de l'analyse du comportement de vote est aussi la dimension temporelle et contextuelle.

Lorsque la confrontation est serrée ou la lutte intense, le rôle des médias croît. Ceux-ci deviennent de vrais arbitres : « Définir un problème comme controverse consacre les médias comme la source principale d'information concernant le thème respectif » (McQuail, Windhal, 200I : 35). C'est toujours la recherche théorique qui montre que l'effet principal des médias est de consolider des opinions et des comportements déjà existants. Les individus sélectionnent, parmi la multitude des moyens d'informations, ceux qui correspondent à leurs croyances et opinions. Ainsi, les citoyens renforcent-ils leurs propres options.

Les quatre campagnes électorales que les Roumains ont connues pendant les dix dernières années permettent une analyse des résultats et des possibles jugements de valeur. Pourtant, on peut remarquer une caractéristique qui leur est commune : l'absence du vote « pour », même si chaque campagne est particulière. La première, organisée seulement cinq mois après la Révolution de décembre 1989, a été marquée par les tensions du moment et a exprimé la naïveté qui accompagne une période de démarrage politique. La deuxième (1992), que l'on peut considérer comme la première du fait qu'elle s'est déroulée après la promulgation de la nouvelle Constitution, a marqué la vraie confrontation entre deux forces politiques de poids comparable. La campagne de 1996 a peut-être été la plus disputée, la plus spectaculaire aussi par ses résultats, et la plus préparée par sa logistique. Enfin, la dernière (2000), ayant 
sans doute un profil moins prononcé que celle qui l'a précédée, est importante par d'autres traits, et par son rapprochement avec des campagnes plus classiques. Elle ressemble à celle de 1996, car le gagnant sera finalement celui qui était dans l'opposition et qui a attaqué la première position, en tant que parti et candidat à la présidence du pays. Cependant, sa structure est complètement différente, ce qui la place dans un autre modèle explicatif.

\section{Une campagne ne peut pas être uniquement regardée par les « yeux» d'une autre}

Une analyse, même sommaire, des campagnes s'étant déroulées dans la période de transition, entre le régime communiste et la période actuelle, reflète l'évidence qu'aucune élection ne ressemble à une autre : des contextes et des problèmes variés, des confrontations particulières se font jour. Ce ne sont pas uniquement les acteurs qui sont différents : les partis et les alliances le sont aussi. De même, les aspects dominants du moment - les messages, les slogans, les programmes et les discours - sont spécifiques. Les alliances et les scores d'une campagne sont souvent induits par les équilibres à l'intérieur d'un espace politique, par la situation politique à un certain moment, mais aussi par la préparation stratégique, par la capacité des « combattants » à répondre à la population, non pas uniquement aux partisans convaincus d'un parti ou d'un candidat.

Prenons l'exemple de la campagne de 1996. Sa caractéristique principale est l'incertitude concernant le gagnant, incertitude qui a plané jusqu'aux derniers moments de la compétition. Cet aspect constitue la dominante de ces élections respectives. On ne peut pas regarder la campagne de 2000 avec les « yeux» de la campagne de 1996, car on risquerait de ne pas la comprendre. Lors des élections de 1996, deux protagonistes importants, de force semblable - Ion Iliescu (président de la Roumanie pendant la période 1990-1996 et, depuis 2000, représentant du Parti démocrate et social roumain) et Emil Constantinescu (président de la Roumanie entre 1996 et 2000, représentant la Convention démocratique roumaine) -, auxquels on pouvait ajouter d'autres formations faisant, elles aussi, de bons scores. Ceci signifie que la population pouvait faire un choix entre deux offres comparables. Dans une situation de ce type, les médias jouent un rôle important, parce qu'ils répondent à un réel besoin de l'électeur de s'informer pour mieux décider.

La campagne de 2000 n'a pas proposé aux Roumains une confrontation où les compétiteurs avaient des chances semblables de gagner. Les acteurs principaux, le Parti démocrate et social roumain, et son représentant dans la course présidentielle, lon lliescu, ne se sont pas affrontés pour ne pas risquer de perdre la compétition. Les faits étant connus, presque décidés dès le début, la campagne n'a pu être spectaculaire. L'imprévu a été l'occupation de la deuxième 
place dans la course présidentielle et parlementaire par Corneliu Vadim Tudor et sa formation, considérée comme extrémiste, le Parti de la grande Roumanie (PRM). Dans ce contexte, la presse n'a pas joué le rôle qu'elle avait joué en 1996. Bref, une campagne à résultat annoncé.

À cette spécificité, il faut ajouter le «portrait » des enjeux politiques des deux campagnes. Sur le plan politique, la campagne de 1996 marque un moment de maturité indiscutable de la société roumaine, prouvé aussi par le transfert sans problèmes et, d'une certaine manière, élégant du pouvoir. L'expérience était prévisible. Elle était accompagnée d'une certaine inquiétude de la part des cercles intérieurs et probablement extérieurs. Aucun reproche concernant l'irrégularité des élections, aucune culpabilité. Tout simplement, le verdict de l'électorat a été accepté et même assumé. Le pouvoir, qui a perdu, a entendu assumer le rôle nouveau de force politique de l'opposition et a essayé de l'exercer.

La campagne de 2000 marque aussi la fin d'une politique excessivement idéologique, d'une stratégie qui avait fait de la politique le critère principal de référence. Le dernier tour des élections présidentielles de 2000 s'est disputé entre Ion Iliescu et Corneliu Vadim Tudor. Redoutant que le deuxième candidat échoue, tous les leaders politiques ont appelé leur électorat à voter lon lliescu. Le paradoxe naît de ce que, jusqu'à ce moment, ces leaders avaient durement critiqué lon lliescu, l'accusant de communisme et de liaisons avec Moscou. L'appel à élire l'actuel président roumain a posé un problème de crédibilité qui explique, en partie, les difficultés que rencontre actuellement l'opposition. La légitimité des personnes placées au premier plan de cette orientation s'en est trouvée profondément affectée. En outre, ce type de politique rencontre des problèmes d'audience et ne peut obtenir l'adhésion de cercles sociaux significatifs. La campagne de 2000 a accordé une place tout à fait spéciale aux approches appliquées, plus détachées des repères idéologiques. Après une décennie de confrontations électorales marquées par des discours passionnés sur l'anticommunisme et la « démocratie originale »', en 2000, les thèmes électoraux deviennent pragmatiques et favorisent les aspects concrets de la période de transition du régime totalitaire à l'économie de marché. Naturellement, les formations tentées par des approches idéologiques, confrontées aux nouvelles demandes, ont dû se réorienter. Bien sûr, un tel processus n'est pas sans provoquer des convulsions intérieures, surtout pendant une période au cours de laquelle la pauvreté progresse. Ceux qui ne tiennent pas compte de cette dimension doivent assumer des risques difficiles à évaluer. À cet égard, les élections de 2000 fonctionnent comme un avertissement.

\footnotetext{
' «La démocratie originale » est le concept électoral prédominant du discours initial d'lon lliescu et de sa formation politique, au début des années 90. Le concept théorise la nécessité d'une approche roumaine de la réalité sociale, politique et économique, différente de l'expérience communiste ou capitaliste occidentale.
} 
Le pragmatisme ${ }^{2}$ présente aussi un autre avantage. II pourra favoriser la création d'un discours pathétique qui promet, sans véritablement tenir compte des moyens de lutter contre une pauvreté déjà chronique. Le discours affirme qu'on rendra justice - et qu'on le fera fermement - pour être crédible et même gagner, en s'appuyant sur le désir naturel et légitime exprimé par certaines tranches de l'électorat. Si la réalité est pauvre, sans perspective, sans espoir, la parole politique, même extrémiste, peut devenir raisonnable et acquérir une forme de légitimité. Dans la vie politique roumaine, le pragmatisme pourra également représenter un antidote pour ce type de discours ; c'est pour cela qu'il est à espérer que les approches pragmatiques et appliquées vont progresser.

\section{6 : pour la première fois, les médias décident}

La campagne de 1996 est importante parce que les médias ont joué un rôle significatif, on pourrait même dire décisif, sur les résultats. Cette particularité est en lien avec celle de la campagne, du contexte, mais aussi avec l'engagement des médias en faveur du « changement 》, visible dès 1992, mais selon d'autres dimensions et caractères d'influence. Selon les sondages de l'Institut de sociologie de l'Académie Roumaine ${ }^{3}$, les médias (la télévision, la radio et la presse écrite - dans cet ordre) ont été les canaux d'information les plus utilisés pendant la campagne électorale de 1996 (Stefanescu, 200 I). Environ 25 \% des électeurs ont reconnu que leur décision a été influencée par les débats télévisés mettant en scène les candidats (émissions suivies par $76 \%$ de l'électorat). Si les médias ont été aussi influents, c'est aussi parce que tous - à l'exception des stations de radio et de télévision nationales et de quelques publications à tirage restreint - se sont mobilisés, non pour la coalition ${ }^{4}$ qui a gagné les élections, mais contre le pouvoir qui finissait son mandat. Et ceci de manière déclarée, sans réserves. Voilà ce que reconnaissait Ion Cristoiu ${ }^{5}$ dans Evenimentul Zilei (22/I I/96) : « J'ai dirigé contre lon lliescu toute l'artillerie publicistique, tant celle du journal, que la mienne personnellement ». Au début de 1996, dans les conclusions d'une émission à ProTV, Mihai Tatulici déclarait : « La seule solution est de choisir d'autres personnes pour gérer les problèmes du pays ».

\footnotetext{
${ }^{2}$ Le pragmatisme, en tant que concept, se réfère à l'influence dominante des valeurs de l'utilité dans le cadre du système des valeurs de la vie. Dans le cas de la campagne électorale de 2000, il s'agit de l'approche qui a favorisé le traitement des thèmes visant les solutions possibles aux problèmes quotidiens des Roumains.

${ }^{3}$ Institut de sociologie de l'Académie roumaine, enquête panel en trois vagues (Iongitudinale), oct.-nov. 1996.

${ }^{4}$ En 1996, plusieurs partis de l'opposition ont formé une alliance électorale contre le PDSR, le parti au pouvoir et, sous le nom de CDR (Convention démocratique roumaine), ont gagné les élections. II s'agit de la coalition formée par le Parti national paysan chrétien et démocrate (PNTCD), le Parti national libéral (PNL), le Parti démocrate (PD) et I'Union démocratique des hongrois de Roumanie (UDMR). ${ }^{5}$ En 1996, le journaliste Ion Cristoiu était le directeur du journal Evenimentul Zilei, l'un des plus importants quotidiens roumains.
} 
Le rôle très important - je dirai essentiel - des médias dans ce qui s'est passé, en novembre 1996, ne résulte pas seulement de l'impact direct des attitudes et des opinions formulées. II montre un vrai mécanisme de modelage de l'opinion, qui a exercé une influence plus profonde, même si elle n'a pas toujours été perceptible. Ce qui caractérise la campagne est le fait que la majorité, si ce n'est la quasi-totalité des moyens de communication de masse, soutenait ouvertement le changement. Le votant, adepte du parti qui allait être écarté du pouvoir - le Parti démocrate et social roumain - c'est-à-dire lon lliescu, trouvait difficilement des soutiens dans le champ médiatique. Ainsi, a-t-on déclenché un processus que l'on peut comprendre si l'on fait appel au paradigme de « la spirale du silence » (Neumann, 1984). L'électorat d'Ion Iliescu a été silencieux, discret et, de toute manière, moins présent. Les partisans d'Emil Constantinescu étaient, au contraire, plus actifs - parfois bruyants - et donnaient l'impression d'une omniprésence, même si, du point de vue numérique, ils se situaient audessous de ceux de l'ex-président. Une grande masse d'indécis, presque un quart du nombre total des électeurs, attendait. Leur choix imposerait la victoire. C'est dans ce contexte particulier que l'influence puissante des médias doit être appréhendée. On peut considérer que les médias jouent le rôle de miroirs immenses, ayant la capacité de projeter une autre image des partis et de leur pouvoir réel. Ils peuvent « diminuer » un parti dans la perception publique, de même que, par la présentation et la fréquence des apparitions, ils peuvent amplifier, avantager et consacrer. II est évident que ceci peut s'accomplir lorsque la marge est étroite, lorsque les pouvoirs réels des forces politiques engagées dans la compétition politique sont assez semblables.

Le plus important est que, dans la campagne de 1996, les médias ont joué le rôle de distributeurs « d'opinions légitimes » ${ }^{6}$. Au moment où ils ont soutenu ouvertement, ou implicitement, les positions d'Emil Constantinescu, les points de vue de ses partisans ont acquis une légitimité publique. Au contraire, ceux qui estimaient que leurs positions étaient différentes, voire opposées aux tendances dominantes, ont de moins en moins été tentés de s'exprimer publiquement. La peur de s'attirer le désaccord de la « majorité » et d'être isolés, a déclenché le processus de « spiralisation ». Nous pensons que le rôle essentiel de la presse, dans ce qui s'est passé en novembre 1996, est intervenu ici. Celle-ci a consacré et élevé au statut « d'opinion dominante » des positions qui ne l'étaient pas. Dès lors, le mécanisme de formation de l'opinion publique a connu une graduation. Ceux qui ont partagé la position définie par la presse ont affirmé, encore plus fermement, leurs points de vue, faisant que leur opinion

\footnotetext{
${ }^{6}$ Si la vie politique post-communiste a déçu les Roumains, les journaux et, plus tard, les radios et les télévisions ont gardé la préférence du public. Les médias ont été perçus comme des informateurs et des lieux d'expression. D'après le Candidate Countries Eurobarometer, en 200I, 65 \% des Roumains ont suivi, chaque jour, les informations à la télévision.

${ }^{7}$ Le concept de « spiralisation » se réfère au phénomène social décrit par la théorie de la spirale du silence (Neumann, 1984) : il s'agit d'une opinion qui peut être exprimée en public, sans risque de sanctions, et sur laquelle peut s'appuyer l'action menée en public.
} 
s'est répandue, même si, en réalité, elle était différente. Au contraire, ceux, probablement plus nombreux, qui ne partageaient pas ces opinions, se sont murés dans un silence plus accentué, pouvant provoquer un sentiment d'incertitude quant à la capacité des individus à juger les événements. Une telle situation entraîne un risque pouvant déclencher un processus de déclin.

En 2000, le Parti démocrate et social roumain a gagné les élections de façon nette. Comment cela s'explique-t-il ? Au moment des élections de 1996, le parti n'avait atteint qu'un faible pourcentage d'opinions favorable (15-16\%), le niveau le plus bas atteint par cette formation. À notre avis, sa progression est le fait de la prestation de la coalition au pouvoir qui a été frappée par d'infinies querelles et par une forme d'instabilité gouvernementale. Par conséquent, le Parti démocrate et social roumain a pu faire valoir ses atouts. Les interminables contestations à l'intérieur de la coalition ont réveillé un désir au sein de la population : celui d'avoir un parti puissant ne rencontrant pas ce type de problèmes. En outre, le parti concentrait une expérience politique et de gouvernement, ainsi qu'une considérable compétence managériale : des atouts qui se situaient « en miroir » par rapport aux déficiences de la coalition au pouvoir.

\section{0 : avec qui le citoyen aurait-il pu voter?}

La campagne de 2000 s'est déroulée dans un contexte différent. Elle présentait, entre autres particularités, une nouvelle identité. C'est pour cela que, si on essayait de « lire » cette campagne à la lumière du paradigme de la « spirale du silence », elle serait incompréhensible. Le paradigme part de l'hypothèse que les gens connaissent l'opinion dominante de leur groupe d'appartenance et que ces perceptions façonnent leur comportement. Autrement dit, s'ils partagent le positionnement de l'opinion dominante (du point de vue symbolique et non pas nécessairement statistique), ils ont davantage de courage pour l'exprimer publiquement. Dans le cas contraire, ils préfèrent le silence. Finalement, ceux qui partagent les opinions dominantes s'expriment activement, tandis que les « minoritaires », par peur d'être sanctionnés par la majorité, décident de se taire. Toutefois, nous avons besoin d'un autre modèle explicatif, qui fixe d'autres règles lors d'une campagne. Nous considérons que, du point de vue théorique, le modèle le plus approprié est celui élaboré par Paul Lazarsfeld, Bernard Berelson et Hazel Gaudet (1952), dont les recherches ont démontré que 2/3 des électeurs décident de leur vote avant le début de la campagne. Ceci prouve que la vraie campagne électorale est représentée par l'intervalle de temps entre les deux périodes d'élections et que la campagne en tant que telle, joue un rôle minime dans la conversion (plutôt celle des indécis).

On ne pourra comprendre ce qui s'est passé en 2000, si l'on ne saisit pas la grande désillusion de l'électorat devant la prestation politique de la coalition. 
Formée de partis de centre-droite (le Parti national paysan chrétien et démocrate et le Parti national libéral) et de centre-gauche (le Parti démocrate), sans homogénéité politique, malgré une modeste expérience de gouvernement, la coalition a obtenu des résultats décevants : le PIB a baissé, à l'instar de presque tous les indicateurs économiques importants. En outre, la coalition a été traversée par des convulsions intérieures faisant, par exemple, que le gouvernement a été conduit par trois Premiers ministres pendant quatre ans. Durant une année, bien qu'il y ait eu des signes de faiblesse, la population a maintenu ses attentes, tout en regardant avec confiance le gouvernement de la coalition. Cela a été l'une des plus longues périodes de grâce qu'a eu, et qu'aura probablement, un gouvernement. Mais, lorsque l'on investit une telle confiance, la déception qui suit est très grande (et pas seulement par des raisons psychologiques). Dans la période 1996-2000, on a constaté une dégradation évidente du niveau de vie, provoquant un décalage frappant entre la promesse politique et la réalisation. Cet écart n'est pas la seule conséquence d'un discours démagogique, mais aussi du manque d'expérience et d'une certaine incapacité - visible surtout pendant les premières années du mandat - de fixer des priorités et d'amener le gouvernement du pays à un certain niveau de performance. II s'agissait donc non seulement de la désillusion, mais, plus grave encore, de l'incapacité des forces de la coalition à assurer une réelle alternative. La sanction de l'électorat est significative. L'échec le plus sévère a été celui du Parti national paysan chrétien et démocrate, en particulier par rapport à son image. Le Parti démocrate et le Parti national libéral ont pourtant réussi à entrer au Parlement, avec quelques points au-dessus de la moyenne, ce qui montre clairement un vote sanction.

Face à cette désillusion et au manque d'alternative, le discours « judiciaire » de Coneliu Vadim Tudor a pu s'imposer. Cependant, après un gouvernement contre-performant, les forces de la coalition se sont séparées au cours de la période pré-électorale ; elles ont eu leurs propres candidats, ce qui a accentué leur fragmentation. En définitive, une question se pose : avec qui a voté le citoyen dans cette campagne? C'est à cause de cette situation que la sérénité de la campagne de 2000 a été possible. Dès lors, elle ne pouvait qu'engendrer la victoire du Parti démocrate et social roumain et de son candidat. En fait, la part de choix du citoyen était modeste et la campagne ne pouvait rien apporter de plus significatif dans la répartition des votes. En outre, étant bien menées du point de vue du rythme, de la diversité et de la modernité, les campagnes du Parti démocrate et social roumain et de lon lliescu n'ont fait preuve d'aucune agressivité. Dans ce contexte, la différence entre la campagne de 1996 et celle de 2000 est énorme, car aucun enjeu ne pesait sur la dernière. Pour autant, on ne peut pas dire que les gens ont voté par conviction. Si l'on se réfère aux scores du Parti démocrate et social roumain, il est clair que le noyau dur de cette formation a agi autrement. L'électorat aurait-il pu se diriger vers d'autres formations? Si oui, lesquelles? Le bon score obtenu par le Parti démocrate et social roumain est aussi la conséquence d'une offre électorale pauvre. Ceci pose 
le problème de l'offre politique, de la crédibilité des messages et de la consistance des stratégies. Ceci oblige aussi à regarder le processus électoral comme une confrontation entre les offres des différents acteurs politiques, mais aussi comme une rencontre entre la demande du citoyen et la proposition des partis. Que se passe-t-il si le citoyen ne trouve pas de réponse à ses propres désirs et attentes? Sur le marché électoral, toute demande ne se traduit pas obligatoirement par l'apparition d'une offre appropriée : on choisit des partis existants, non pas des partis souhaités.

Dans la campagne de 2000, les médias n'ont pas joué le même rôle qu'en 1996 , parce que le contexte a changé. La lutte était plus étroite, le gagnant déjà connu, et le principal parti de l'opposition était perçu comme le vainqueur naturel. La campagne avait donc comme unique but de fixer les dimensions de la victoire. Dans un tel contexte, quelle fonction auraient pu jouer les médias?

\section{On sanctionne l'ancienne équipe ou on en choisit une nouvelle?}

En regardant plus attentivement les élections en Roumanie, un fait devrait nous préoccuper : l'instauration de la règle du vote négatif. Les recherches montrent qu'en 1996 et en 2000, le citoyen a voté autant pour ceux qui sont venus au pouvoir, que contre ceux qui avaient gouverné jusqu'alors. Dans la décision de vote, ce qui semble compter est la sanction, plus que la confiance. Évidemment, cette situation ne peut être limitée aux faibles performances des gouvernements précédents. Le vote négatif exprime une souffrance, une protestation. Lorsque la qualité de la gouvernance fait défaut, le vote négatif accomplit une nécessaire fonction sanitaire : sanctionner et éloigner des leviers du pouvoir l'amateurisme, l'incapacité et l'incompétence. II décourage le dilettantisme. II prouve que l'opinion publique est capable d'évoluer, et qu'elle ne peut être facilement trompée. La prépondérance du vote négatif fait du mandat un pouvoir qui ne dure pas plus de quatre ans, bien que les gouvernements puissent être modifiés plusieurs fois au cours d'un même mandat. Un tel changement ne permet pas d'accomplir des objectifs à long terme ; il introduit un type d'évolution saccadée, marquée par la durée du cycle électoral. Dans les démocraties matures, on rencontre ces situations lorsque la rotation du pouvoir se fait après une période de quatre ans. Dans ce cas, soit on considère qu'il y a eu une prestation modeste (par exemple, le mandat de Jimmy Carter), soit on a affaire à la clôture d'un cycle historique (à notre avis, la perte des élections par George Bush, en 1992, n'est pas tant due au manque de performance, qu'au fait qu'une étape historique s'achevait, appelant un autre personnel politique). La « normalité » est représentée par deux mandats consécutifs, ce qui signifie huit ans, et une période assez grande pour conduire 
l'action à la lumière de la même plate-forme électorale, de la même stratégie, avec une équipe dont les membres partagent les mêmes convictions et des approches similaires (par exemple, en France, François Mitterrand et Jacques Chirac ont bénéficié tous les deux de deux mandats). II est intéressant de signaler que le vote négatif prépare un autre vote, presque identique, au tour suivant. Comme l'acteur au pouvoir n'y est pas par ses propres mérites, mais à la suite des fautes des autres, il est probable que la faible performance se perpétue. Le fonctionnement de ce vote montre clairement que les raisons du ressentiment envers le pouvoir qui vient de finir son mandat sont grandes, et que les raisons d'optimisme - liées au nouveau pouvoir - sont réduites. II est donc impossible d'avoir un vote «pour ». En ce sens, bénéficier de deux victoires électorales consécutives serait un signe de maturité de la vie politique roumaine; le transfert du pouvoir de 1996 serait le signe d'un retour à la normale qui laisserait place à l'espoir, et dont la récompense ne serait que le vote du citoyen. Toutefois, le ressort de la décision se trouve entre les mains de la classe politique dont dépendent la performance, le développement économique et l'émergence de la prospérité, qui pourraient convaincre de voter « pour ».

\section{La prospérité comme argument de la légitimité}

Nous voudrions insister, ici, sur un autre aspect qui n'a pas de lien direct avec les campagnes électorales, mais qui marquera les stratégies électorales et politiques du pays. On s'est habitué à l'idée que le vote et les processus de choix sont la source exclusive de la légitimité. Le parti qui gagne les élections acquiert une légitimité pour exercer son pouvoir durant un mandat. La source démocratique de la légitimité a été, et reste toujours, essentielle. On ne peut pas parler de légitimité hors des mécanismes démocratiques, hors de la liberté d'exercer les prérogatives démocratiques.

La source démocratique de la légitimité est fondamentale, mais insuffisante. Un parti peut gagner des élections, ayant ainsi et sans contestation, la possibilité de gouverner. Néanmoins, une telle source a besoin d'être accompagnée d'une autre : un mode de gouvernement qui assure et engendre de la prospérité. À condition d'être ensemble, ces deux facteurs peuvent assurer une légitimité entière et incontestable. Si la performance économique manque et que la pauvreté s'amplifie, la légitimité démocratique est en crise. Si les élections se sont déroulées en bonne et due forme, mais que la population vit difficilement, le parti gagnant sera de plus en plus contesté. Un tel processus est important selon deux perspectives. Premièrement, la pauvreté de la Roumanie s'est amplifiée de façon inquiétante, érodant la confiance du citoyen dans le mécanisme démocratique qui doit, ou au moins devrait, privilégier la 
compétence. Le citoyen est alors en mesure de se demander à quoi servent les élections. Le processus démocratique des élections peut-il encore attribuer une légitimité au cas où le gouvernement n'est pas performant et ne prévoit aucune prospérité ? Lorsqu'une telle question survient, de manière explicite ou non, elle déclenche un phénomène de sous-évaluation du processus électoral et des mécanismes démocratiques. Deuxièmement, il s'agit du risque que représente la pauvreté pour la modification du comportement politique et électoral de l'opinion publique. C'est un problème qui est d'ordinaire traité superficiellement, par des phrases stéréotypées, bien que l'histoire nous montre que c'est la pauvreté qui a provoqué les changements politiques les plus spectaculaires, surtout en direction des régimes totalitaires. La pauvreté radicalise une impatience palpable ; elle conduit à voter sous la pression de facteurs différents de ceux d'une situation normale. Par exemple, elle amène à faire des promesses inconsidérées qui deviennent une sorte de drogue et empêchent de supporter les frustrations. Cette vulnérabilité favorise l'apparition de préoccupations et de stratégies de manipulation des esprits. II ne faut pas s'imaginer qu'il existerait des réticences ou une certaine timidité à utiliser de telles stratégies.

L'électorat « achète » finalement ce qu'il trouve et, surtout, ce qui lui convient le plus. La Convention démocratique roumaine a perdu les élections de 2000, faute de discours pragmatique approprié aux besoins psychologiques d'un électorat paupérisé. En 1996, le pouvoir sortant avait considérablement perdu de sa crédibilité, sur fond d'une crise sociale et économique marquée par de graves problèmes tels que la pauvreté, le chômage, l'inflation ou la corruption. Ainsi, le changement du pouvoir a-t-il été déterminé par un double phénomène : d'un côté, le vote de sanction du gouvernement et donc de rejet ; de l'autre, le parti pris des indécis. Les deux phénomènes ont eu comme point de départ la déception et la frustration, déterminées par le contexte problématique des élections. La situation s'est reproduite en 2000 et cette fois, les perdants ont été les gagnants de 1996. Si au cours de la période 1990-1992, on a voté plutôt émotionnellement (contre les anciens communistes ou pour les candidats issus de la révolution de 1989), à partir de 1996, les Roumains ont choisi les opposants de ceux qui les avaient déçus. Pendant les deux premières élections, on a voté contre quelqu'un. Après, on a sanctionné par le vote - donc, toujours « contre ». Finalement, on attend encore que les Roumains donnent leur vote « pour $»$.

Texte révisé par Stéphanie Hurez, CREM, université de Metz Béatrice Fleury-Vilatte et Jacques Walter 


\section{Références}

Almond G., Verba S., 1996, La culture civique. Attitudes politiques et démocratie pour cinq nations, Bucarest, Éd. du Style.

Katz E., 1990, « À propos des médias et de leurs effets », pp. 275-282, in : L. Sfez, G. Coutlée, dirs, Technologies et symboliques de la communication, Grenoble, Presses universitaires de Grenoble.

Lazarsfeld P., Berelson B., Gaudet H., 1952, The People's Choice. How the Voter Makes Up His Mind in the Presidential Campaign, New York, Columbia University Press.

McQuail D., Windhal S., 200I, Modele ale comunicarii [Modèles de la communication], Bucuresti, Comunicare.ro.

Neumann E.-N., 1984, The Spiral of Silence. Public Opinion, Our Social Skin, Chicago, The University of Chicago Press.

Stefanescu S., 200I, « La mobilité des options politiques et les débats télévisés. Une comparaison entre les années électorales 1996 et $2000 »$, La Revue de communication et relations publiques, 4-5, Bucarest.

Vlasceanu L., Miroiu A., 200I, Democratia ca proces. Alegerile 2000 [La démocratie comme processus. Les élections de 2000], Bucuresti, Trei. 